\title{
La formation des maîtres au Brésil
}

Histoire et recherches en cours

The Training of Teachers in Brazil, between History and Historical Object

Die Grundschullehrerausbildung in Brasilien, zwischen Geschichte und

Geschichtsbetrachtung

La formación de los maestros en Brasil, entre historia y objeto de historia

\section{Maria Helena Camara Bastos}

\section{OpenEdition}

Journals

Édition électronique

URL : https://journals.openedition.org/histoire-education/2034

DOI : $10.4000 /$ histoire-education.2034

ISSN : 2102-5452

Éditeur

ENS Éditions

Édition imprimée

Date de publication : 1 juillet 2009

Pagination : 107-124

ISSN : 0221-6280

Référence électronique

Maria Helena Camara Bastos, "La formation des maîtres au Brésil », Histoire de l'éducation [En ligne], 123 | 2009, mis en ligne le 01 janvier 2014, consulté le 20 mai 2021. URL : http://

journals.openedition.org/histoire-education/2034; DOI : https://doi.org/10.4000/histoire-education. 2034

(c) Tous droits réservés 


\title{
La formation des maîtres au Brésil
}

\author{
Histoire et recherches en cours*
}

\author{
Maria Helena CAMARA BASTOS
}

En mai 2004, sous l'égide de l'UNESCO et du ministère de l'Éducation, l'Instituto Nacional de Estudos e Pesquisas Educacionais (INEP) publiait les résultats d'une recherche intitulée "Profil des professeurs brésiliens : ce qu'ils font, ce qu'ils pensent, ce à quoi ils aspirent... ". Dans la préface, Juan Carlos Tedesco ${ }^{1}$ souligne " la forte revalorisation du rôle et de l'importance des enseignants dans les stratégies de réforme éducative qui, pour affronter les défis posés par l'enseignement, doivent viser des politiques globales, dépassant les perspectives sectorielles traditionnellement adoptées. Ces politiques globales revêtent au moins trois grandes dimensions : des actions pour améliorer le profil des aspirants à l'exercice de la profession d'enseignant ; des stratégies pour élever la qualité de la formation initiale des maitres et professeurs, garantir une formation continue tout au long de leur service ; l'établissement de cadres de la carrière enseignante permettant de promouvoir les maitres sans leur faire abandonner la salle de classe [...]. Élaborer ces politiques exige une connaissance fine de ce qu'ont été, dans le passé, non seulement les conditions matérielles du travail des enseignants et les caractéristiques de leur formation, mais aussi les représentations qu'ils avaient de leur profession, du processus

\footnotetext{
* Une première version de ce texte a été présentée au séminaire du Service d'histoire de l'éducation en mai 2005.

1 Perfil dos professores brasileiros: o que fazem, o que pensam, o que almejam..., Pesquisa Nacional UNESCO, São Paulo, Moderna, 2004, p. 12.
} 
de réforme et de leurs élèves, ainsi que de leur idée du civisme et de leurs consommations culturelles".

Au moment où paraissait cette recherche, la population scolaire brésilienne, de la maternelle aux premiers cycles de l'enseignement supérieur (graduação), s'élevait à près de 57,7 millions d'élèves. Il y avait alors, pour former plus d'un million d'étudiants ${ }^{2}, 5880$ cours supérieurs préparant à une licence, dont 3116 relevant du secteur public. À leur côté existaient encore, même si leur fermeture progressive était prévue, des écoles qui menaient au magistério, niveau de fin d'études secondaires autorisant à enseigner : elles étaient 2641 , dont 2050 écoles publiques. Devenir professeur d'école (en maternelle et dans les premières classes de l'enseignement fondamental) n'était guère attractif en termes de traitement et de conditions de travail : la durée hebdomadaire de classe est de vingt heures pour les enfants, mais la majorité des enseignants a deux ou trois classes (le matin, l'après-midi, et un cours du soir), et les différences sont considérables entre les réseaux d'enseignement (privé/public) et entre les différentes régions du Brésil en matière de salaire, d'avantages sociaux et de moyens pédagogiques. En 2004, le professorat restait la profession la moins bien rémunérée à diplôme égal, et l'une des plus massivement féminisées.

Quel était alors le niveau d'étude des professeurs ? 2,2 millions étaient en activité dans le pays, dont 48 \% n'avaient pas de diplôme de niveau supérieur, surtout au niveau élémentaire et préscolaire et dans le Nord et le Nordeste. Cela ne doit pas masquer les évolutions en cours : selon le ministère, en 1999-2000, 350000 professeurs en exercice (environ $16 \%$ du corps) n'avaient pas le niveau de formation requis et $10 \%$ n'étaient même pas allés jusqu'au terme du cursus obligatoire, c'est-à-dire au niveau de la classe de troisième. Cependant, de 1996 à 2000, les maîtres possédant une qualification universitaire étaient passés de $47 \%$ à $52 \%{ }^{3}$.

Comment comprendre la situation actuelle et l'évolution en cours sans revenir sur l'héritage du passé ? C'est ce que nous nous proposons de faire avant de dresser un tableau des recherches en cours sur cette histoire.

2 Très exactement, 1059385 étudiants en 2002.

3 Marco Aurelio Weissheimer, "Privatização e a herança da era FHC ", Adverso, Porto Alegre, 2 quinzena de dezembro de 2001, p. 6-7. 


\section{I - La formation des maîtres dans l'histoire scolaire brésilienne}

Au temps du Brésil esclavagiste, "la maîtrise de l'alphabet, réservée à quelques-uns, a servi de ligne de partage entre la culture officielle et la vie populaire. Le quotidien populaire colonial s'est organisé et reproduit au seuil de l'écrit " ${ }^{4}$. Au moment de l'Indépendance (1822), il existe " une île de lettrés sur une mer d'analphabètes ${ }^{5}$. Cette situation s'est perpétuée jusqu'au début du $\mathrm{XX}^{\mathrm{e}}$ siècle, avec une population analphabète à $80 \%$.

Cependant, on peut distinguer deux périodes, celle des missions et de la catéchisation religieuse, pendant laquelle la Compagnie de Jésus est très active avant d'être expulsée en 1759 du Brésil comme du Portugal, puis la période de Pombal, qui vise à donner au Portugal un système d'enseignement public et uniforme, destiné à instruire une administration plus moderne, réforme toutefois restée sans grand effet sur le Brésil.

La réglementation de la formation des professeurs apparaît avec l'Indépendance. Le décret sur les écoles primaires du 15 octobre 1827 est la première législation sur l'instruction publique nationale. Il propose la création d'écoles primaires " dans tous les endroits peuplés où il leur sera possible de s'établir ", ainsi que l'adoption de la méthode lancastérienne, qui permet d'enseigner à des masses d'enfants de façon économique :

Les écoles seront d'enseignement mutuel [...]. Les professeurs qui n'auraient pas les connaissances nécessaires à cet enseignement iront s'instruire pendant une courte durée et à leurs frais dans les écoles des capitales [des États brésiliens]. Les professeurs enseigneront la lecture, l'écriture, les quatre opérations d'arithmétique, la pratique des fractions, les décimales et les proportions, les notions les plus générales de géométrie pratique, la grammaire de la langue nationale, les principes de la morale chrétienne et la doctrine de la religion catholique et apostolique romaine, à la mesure de la compréhension des enfants, en donnant la préférence, pour l'enseignement de la lecture, à la Constitution de l'Empire et à l'histoire du Brésil [...] ; ils enseigneront également l'économie domestique ${ }^{6}$.

4 Alfredo Bosi, A dialética da colonização, São Paulo, Cia das Letras, 1995.

5 José Murilo Carvalho, A construção da ordem, Rio de Janeiro, Campus, 1981.

6 Primitivo Moacyr, A instrução e o Império. Subsídios para a história da educação no Brasil (1823-1835), São Paulo, Nacional, 1936. 
L'introduction de l'enseignement mutuel au Brésil avait précédé ce décret En 1820, le gouvernement autorise João Batista de Queiroz à partir apprendre la méthode lancastérienne en Angleterre. En 1822, le Bureau des affaires de la guerre prend sous sa responsabilité l'école mutuelle de Rio de Janeiro, puisque des militaires semblent particulièrement aptes à assumer un enseignement consistant à donner des ordres et à veiller à leur bonne exécution par les élèves. Il faut attendre le 12 mai 1837 pour que l'Empire rende les deux fonctions incompatibles.

C'est pour former les professeurs à cette méthode anglaise qu'est créée la première école normale du Brésil (1835-1851), à Niterói (province de Rio de Janeiro). Pour y être admis, le candidat doit être " citoyen brésilien, âgé de plus de dix-huit ans, de bonnes mœurs ; et sachant lire et écrire " 8 . Ce mouvement en faveur du système mutuel a été très actif en France sous la Restauration et le premier ouvrage pédagogique publié au Brésil est celui du baron de Gérando, intitulé Cours normal pour les professeurs du primaire, ou directions relatives à l'éducation physique, morale et intellectuelle dans les écoles primaires, édité à Paris en 1832. Il met l'accent sur l'éducation morale bien plus que sur la transmission des connaissances, de façon à former un élève et futur citoyen docile et discipliné. Comme l'école catholique, l'école laïcisée vise explicitement à contrôler les individus et les mœurs sociales.

Les examens de recrutement pour l'enseignement public comprennent "la lecture et l'analyse grammaticale d'un discours en prose et d'un autre en vers ; la dictée ; la pratique des quatre opérations d'arithmétique sur les nombres entiers, les décimales et les fractions, et la résolution de problèmes qui demandent l'emploi des proportions ; la résolution de problèmes généraux de géométrie ; la résolution de problèmes géographiques sur le globe et la sphère, la description de la terre, la présentation politique et géographique des différents États ; l'exposé succinct des principes de la doctrine chrétienne, tant morale que dogmatique ; enfin, la manière de les transmettre grâce à la méthode lancastérienne ${ }^{9}$.

7 Maria Helena Camara Bastos, "A formação de professores para o ensino mútuo no Brasil: o curso normal para professores de primeiras letras do Barão de Gérando (1839) » in M. H. Camara Bastos, L. M. de Faria Filho (dir.), A Escola Elementar no século XIX: o método monitorial/mútuo, Passo Fundo, Ediupf, 1999.

8 Heloísa Villela, " A primeira Escola Normal do Brasil " in Clarice Nunes (dir.), O passado sempre presente, São Paulo, Cortez, 1992, p. 29.

9 Heloísa Villela, "O ensino mútuo na origem da primeira escola normal do Brasil " in M. H. Camara Bastos, L. M. de Faria Filho (dir.), A escola elementar no século XIX ..., op. cit., p. 162. 
On compte six écoles normales en 1860, et en 1876 le gouvernement impérial en crée deux autres, destinées à devenir des établissements modèles. Elles fusionnent en 1879 pour constituer une seule école normale mixte, dont le curriculum, très ambitieux, est de six ans. On insiste sur la "méthode intuitive " ${ }^{10}$ l'enseignement "par l'aspect " (la leçon de chose), qui constitue alors la "pédagogie réaliste " en vogue dans les pays industrialisés. Le gouvernement impérial, ainsi que des acteurs privés, favorisent les conférences populaires, les conférences pédagogiques, les expositions scolaires, les congrès de l'instruction publique, la création du Musée scolaire national et de bibliothèques scolaires, toutes initiatives qui visent à stimuler le débat pédagogique et à renouveler les pratiques enseignantes dans une sorte de "formation continue " ${ }^{11}$. Ces initiatives ont cependant une portée géographique limitée, car les candidats capables sont peu nombreux, au point que la tutelle de l'État paraît un frein plus qu'une aide au recrutement. La réforme de Leôncio de Carvalho de 1879, pariant sur la liberté de l'enseignement, ouvre l'exercice professionnel aux non-diplômés : "Peuvent enseigner tous ceux qui s'y jugent habilités, sans dépendre d'épreuves officielles d'aptitude ou d'une autorisation préalable ; et que chaque professeur soit autorisé à exposer librement ses idées et à enseigner les doctrines qu'il tient pour vraies, par la méthode qu'il juge la meilleure "12.

Après la proclamation de la République (1889), les écoles normales se multiplient rapidement. Le Pedagogium est créé en 1890 sur le modèle du Musée pédagogique français ${ }^{13}$. C'est un centre qui "impulse les réformes et améliorations dont l'instruction nationale a besoin, offre aux professeurs du public et du privé les moyens d'instruction professionnelle qui pourraient leur manquer, la description des meilleures méthodes d'enseignement et du matériel d'enseignement le plus perfectionné " : exposition permanente au Musée pédagogique ; conférences et cours scientifiques ; cabinets et laboratoires de sciences physiques et naturelles ; concours ; expositions scolaires annuelles ; école primaire modèle ; classe-modèle de dessin et atelier de travaux manuels ; organisation de collections modèles pour l'enseignement scientifique dans les écoles publiques ; publication d'une revue pédagogique. Ce dispositif dure

10 Analete R. Schelbauer, A constituição do método intuitivo na Província de São Paulo (1870-1889), São Paulo, Feusp, 2003 (thèse).

11 Maria Helena Camara Bastos, Pro Patria Laboremus. Joaquim José de Menezes Vieira (1848-1897). Bragança Paulista: USF, 2002.

12 Iria Brzezinki, Pedagogia, pedagogos e formação de professores, Campinas, Papirus, 1996.

13 Maria Helena Camara Bastos, Pro Patria Laboremus, op cit. 
presque trente ans (de 1890 à 1919), et l'idée sera reprise en 1937 avec l'INEP, sous le ministère Capanema.

Dans cette période de grands changements économiques (industrialisation, développement urbain, immigration), l'éducation est vue comme un pilier du progrès national (Nagle ${ }^{14}$, Carvalho ${ }^{15}$ ). En 1925, la réforme Rocha Vaz instaure l'obligation du diplôme de l'école normale pour professer dans l'enseignement élémentaire (6-10 ans), et une préparation en trois ans, dans des gymnases complémentaires à caractère pratique et professionnel, pour former ceux qui enseigneront dans les quatre années suivantes (l'équivalent de l'enseignement primaire supérieur français). Cette formation, qui durera jusqu'en 1946, attire rapidement les jeunes filles de bonne famille, qui trouvent là une instruction générale et pratique qui leur convient, qu'elles aient ou non l'intention d'enseigner. Mariza Corrêa ${ }^{16}$ parle de la "révolution des normaliennes " : en 1933, $85 \%$ du corps enseignant primaire est constitué de femmes ; en 1944, $90 \%$; en $1958,93 \%$.

C'est l'époque où la référence devient l'école nouvelle, escola nova, d'où le nom d'escolanovistes donné à ceux qui partagent " enthousiasme pour l'éducation " et " optimisme éducatif ", selon la formule de Jorge Nagle, l'un des premiers à avoir fait l'histoire de cette époque ${ }^{17}$. Ces idées pénètrent l'Association brésilienne d'éducation (ABE), créée en $1924^{18}$ et, lors de la première Conférence nationale d'éducation, tenue à Curitiba en 1927, on envisage de créer des écoles normales supérieures et un professorat primaire unifié national. Les Pionniers de l'éducation nouvelle, dont le Manifeste paraît en mars 1932, accèdent aux responsabilités politiques et fondent nombre d'institutions scolaires destinées à servir d'écoles modèles pour la formation des maîtres.

L'ère Vargas qui débute en 1930, centraliste, autoritaire, corporatiste, met fin au régime parlementaire et modernise le pays, tout en soutenant l'idée d'une culture brésilienne et d'une histoire nationale. Ministre de l'Éducation de 1934 à 1945, Capanema crée en 1937 l'Institut national de pédagogie (INEP), à la fois organisme de recherche et de diffusion de la recherche ou des expériences

14 Jorge Nagle, Educação e sociedade na Primeira República, São Paulo, EPU/Edusp, 1974.

15 Marta M. C. de Carvalho, A escola e a República, São Paulo, Brasiliense, 1989.

16 Mariza Corrêa, "A revolução das normalistas ", Cadernos de Pesquisa, São Paulo (66), 16-27, ago 1988.

17 Jorge Nagle, Educação e sociedade ..., op. cit., p. 99-100.

18 Marta M. C. de Carvalho, Molde nacional e forma cívica: higiene, moral e trabalho no projeto da Associação Brasileira de Educação (1924-1931), Bragança Paulista, USF, 1999. 
pédagogiques, puisqu'il doit " recueillir, systématiser et diffuser les travaux réalisés par les institutions pédagogiques, publiques et privées. En outre, il lui incombera de promouvoir des échanges soutenus sur les recherches relatives à l'éducation avec les autres nations où ce problème est l'objet d'un soin particulier de la part des pouvoirs publics ou des entités privées ". Dirigé par Lourenço Filho, l'INEP reprend ainsi les fonctions du Pedagogium : publications, recherches, études et perfectionnement des professeurs ${ }^{19}$.

Durant cette période, se créent nombre de dispositifs parallèles pour former les professeurs et élever leur niveau de qualification. Le cours de pédagogie établi dans les facultés est destiné aux étudiants visant le professorat dans l'enseignement secondaire ou les écoles normales ${ }^{20}$. Cependant, même s'il est prévu de considérer les instituts d'éducation comme partie intégrante de l'université, " les facultés d'éducation, bien que créées par loi depuis 1931, ne vont s'installer que tardivement, avec la réforme universitaire de $1968 "^{21}$. L'école pionnière dans l'élévation des études pédagogiques au niveau universitaire a été l'École de professeurs de l'Institut d'éducation de Rio de Janeiro, qui a été incorporée à l'Université du district fédéral (1935-1938) et offre deux années de formation " supérieure " au-delà du baccalauréat.

Lorsque la première université du Brésil (celle de Sao Paulo) crée une faculté de philosophie en 1939, elle inclut parmi ses objectifs " la préparation des candidats au professorat de l'enseignement universitaire et normal ", avec les sections suivantes : philosophie, sciences (cours de mathématique, physique, chimie, histoire naturelle, géographie et histoire, sciences sociales, dessin à l'École des beaux-arts), lettres (cours de lettres classiques, lettres néo-latines, lettres anglo-germaniques), pédagogie, didactique. Ces cours conféraient le diplôme de "bachelier " (de niveau universitaire, obtenu en trois ans) et de licencié (après une année supplémentaire consacrée à la didactique, générale et spécialisée, et à des cours de biologie et psychologie de l'enfant, de sociologie de l'éducation et d'administration scolaire), cursus dit " $3+1$ ". L'objectif était de " préparer les travailleurs intellectuels à l'exercice de hautes activités culturelles, en tant qu'amateurs ou professionnels ". Cependant, le statut de

19 J. G. Gondra, "O veículo de circulação da pedagogia oficial da República: A Revista Pedagógica ", Revista Brasileira de Estudos Pedagógicos, Brasília, v. 78, n 188,189,190, jan/dez. 1997, p. 374-395.

20 Olinda Evangelista, A formação universitária do professor. O Instituto de Educação da Universidade de São Paulo (1934-1938), Florianópolis, NUP/CED/UFSC/Cidade Futura, 2002.

21 Iria Brzezinki, Pedagogia, pedagogos e formação..., op. cit. 
"pédagogue " créé par ce décret reste flou : doit-il fournir des spécialistes en éducation pour le ministère de l'Éducation et/ou des professeurs spécialisés pour les écoles normales ${ }^{22}$ ?

La Loi organique sur l'enseignement normal (1946) instaure, à côté des écoles normales, des écoles régionales appelées instituts d'éducation pour former des instituteurs et des professeurs. Ces instituts offrent des cours de spécialisation pour les enseignants du primaire, appelés cours post-normaux $^{23}$, à l'intention des futurs directeurs, inspecteurs, orienteurs, statisticiens et évaluateurs. En 1949, on comptait environ 540 écoles normales au Brésil, mais $40 \%$ des enseignants du primaire n'étaient pas passés par ces institutions.

Cependant, à partir de 1945, la "redémocratisation du pays " met la question de l'école publique à l'ordre du jour, et le débat s'engage sur la première loi sur les directives et bases de l'éducation nationale (LDB), finalement votée en 1961. O Manifesto dos Educadores Mais Uma Vez Convocados (Le Manifeste des éducateurs convoqués encore une fois, 1959), la création de différentes associations de professeurs du primaire - germes des syndicats de professeurs d'aujourd'hui - marquent l'effervescence de l'époque. La demande sociale d'éducation entraîne la création désordonnée d'universités et d'instituts d'enseignement supérieur, publics et privés. Dans les années 1960, établir une "faculté de philosophie " était facile : on en compte alors 113, alors que le nombre d'universités ne dépasse pas 31 .

En 1962, la réforme inspirée par Valmir Chagas modifie le cours de pédagogie, ce qui allonge le cursus de formation pour les professeurs du primaire (graduação) et pour les "pédagogues " (les spécialistes de l'éducation doivent avoir le niveau de pós-graduação). Ce cours, ouvert aux bacheliers et licenciés de toute provenance ${ }^{24}$, est destiné à former les " techniciens en éducation " ou les "spécialistes en éducation ", ainsi que les professeurs de sciences de

22 Carmem Silvia B. Silva, Curso de Pedagogia no Brasil. História e identidade, São Paulo, Autores Associados, 1999.

23 Ce sont des cours d'éducation préscolaire, d'enseignement primaire complémentaire, de dessin, de musique et d'administration scolaire.

24 Carmem Silvia B. Silva, Curso de Pedagogia no Brasil ..., op. cit. 
l'éducation du cours normal, grâce au baccalauréat ${ }^{25}$ et à la licence. Consistant en un socle commun de matières générales (sociologie générale, sociologie de l'éducation, psychologie de l'éducation, histoire de l'éducation, philosophie de l'éducation et didactique), il reste en vigueur pendant presque trente ans.

C'est la réforme universitaire de 1968 qui crée des facultés d'éducation autonomes, indépendantes des facultés de philosophie, de sciences et de lettres, pour former les professeurs et spécialistes en éducation ${ }^{26}$. Des cours de pós-graduação sont créés - lato et stricto sensu - pour stimuler la recherche, former des spécialistes en hautes études pédagogiques et fournir des candidats à l'enseignement supérieur. En 1996, la nouvelle LDB prévoit la suppression des écoles normales : tous les professeurs, primaires et secondaires, seront à terme recrutés au niveau supérieur. Pour l'heure, les écoles normales, qui donnaient une instruction secondaire à des adolescents d'origine généralement populaire, sont transformées en "instituts supérieurs de l'éducation ", c'est-àdire en écoles professionnelles préparant ceux qui n'ont pu aller à l'Université à enseigner à l'école maternelle et élémentaire. Ils dispensent aussi une formation pédagogique pour les étudiants diplômés qui choisiraient l'éducation de base après leur cursus universitaire. Enfin, ils assurent la formation continue des professionnels de l'éducation.

En 2001, le Conseil national d'éducation définit pour l'ensemble du Brésil un cursus de formation des enseignants du primaire, qui doivent désormais obtenir une licence. Le cours de pédagogie doit comporter dans son cursus un noyau de contenus de base articulant théorie et pratique. La " théorie ", ce sont " les fondements philosophiques, historiques, politiques, économiques, sociologiques, psychologiques et anthropologiques nécessaires à la réflexion critique dans les divers secteurs de l'éducation de la société contemporaine ". La formation reste donc très académique, et l'initiation pratique au métier n'est pas prioritaire.

25 Pour le baccalauréat, le cursus comprend sept matières, cinq obligatoires (psychologie de l'éducation, sociologie générale et de l'éducation, histoire de l'éducation, philosophie de l'éducation, administration scolaire), et deux optionnelles (biologie, histoire de la philosophie, statistique, méthodes et techniques de recherche pédagogique, culture brésilienne, éducation comparée, hygiène scolaire, cursus et programmes, techniques d'éducation audiovisuelles, théorie et pratique de l'école secondaire, introduction à l'orientation) (voir Carmem Silvia B. Silva, Curso de Pedagogia no Brasil ..., op. cit., p. 37).

26 On distingue la licence pleine (en quatre ans), la licence courte (en deux), l'administration scolaire, l'inspection scolaire, l'orientation scolaire, la supervision scolaire, l'éducation spécialisée, les disciplines pédagogiques pour l'école normale. 
Pour résumer, jusqu'à la mi-XX $\mathrm{XX}^{\mathrm{e}}$ siècle, la formation des professeurs s'est confondue avec une instruction de niveau secondaire, dans des écoles normales - publiques ou privées, laïques ou religieuses - réservées aux futurs instituteurs des écoles primaires. À partir des années 1930, les professeurs du second cycle du secondaire doivent acquérir des grades universitaires (une licence). Les grandes lignes de l'histoire politique et institutionnelle de la formation des maîtres étant désormais solidement tracées, la recherche a considérablement enrichi la connaissance que nous avons aujourd'hui des processus et des acteurs de cette histoire.

\section{II - La recherche sur l'histoire de la formation des maîtres au Brésil}

La recherche brésilienne s'inscrit volontiers au sein de réseaux organisés. Parmi ces réseaux, on citera le Groupe de travail (GT) en histoire de l'éducation, créé en 1984 au sein de l'Association nationale de recherche en éducation (ANPEd) ; le groupe Histoire, société et éducation au Brésil (HISTEDBR), dirigé par Dermeval Saviani, qui coordonne depuis 1986 d'autres groupes de recherche dans divers États brésiliens en organisant des rencontres et en encourageant la collecte et le catalogage des sources de l'éducation brésilienne ${ }^{27}$; la Société brésilienne d'histoire de l'éducation, fondée en 1999, membre de l'International Standing Conference for the History of Education (ISCHE) depuis $2000^{28}$. Une autre initiative est la constitution de groupes de recherche dans les programmes de pós-graduação ${ }^{29}$, qui a dynamisé la publication de sources en histoire de l'éducation. En particulier, la revue de l'Association du Rio Grande do Sul de chercheurs en histoire de l'éducation est un espace privilégié de diffusion des

27 Dermeval Saviani, José C. Lombardi, "Grupo de Estudos e Pesquisas História, Sociedade e Educação no Brasil (HISTEDBR): histórico e situação atual ", Educação em revista, Belo Horizonte, n 34, 2001, p. 135-146.

28 Depuis 2007, les sociétés nationales sont reconnues par l'ISCHE en tant que sociétés associées (Ndlr).

29 La première thèse sur l'histoire de l'éducation brésilienne, soutenue par José Querino Ribeiro à l'Université de São Paulo en 1943, est intitulée Ensaios sobre a significação e a importância da memória sobre a reforma dos estudos na Capitania de São Paulo, escrita em 1816 por de Martim Francisco Ribeiro de Andrade Machado. 
recherches et d'importants débats historiographiques ${ }^{30}$. Á signaler aussi, la constitution de Centres de mémoire de l'éducation dans divers États brésiliens.

De nombreux congrès nationaux et internationaux ${ }^{31}$ ont permis aux chercheurs brésiliens de rencontrer leurs collègues et de mieux diffuser leurs recherches, grâce à des périodiques spécialisés : Revista História da Educação, Revista Brasileira de História da Educação, Cadernos de História da Educação, la revue électronique de l'HISTEDBR. Il faut également signaler l'existence de collections éditoriales consacrées à ces questions ${ }^{32}$. Les programmes de pós-graduação au Brésil, les stages de formation et de recherche à l'étranger sont des espaces privilégiés de construction et d'élargissement du champ.

\section{1 - Une historiographie déséquilibrée et lacunaire}

Il n'existe pas d'étude systématique sur la situation de la recherche concernant l'histoire de la formation enseignante et/ou la profession enseignante au Brésil. Par la richesse et la diversité de ses approches, la polysémie du terme formation du professeur (" professeur " désignant tout enseignant, quel que soit le niveau auquel il intervient) rend difficile une analyse systématique de l'état de la connaissance. La recherche privilégie le professeur en fonction plutôt que le futur professeur, l'étudiant. Une autre difficulté réside dans la dispersion des lieux de recherche, du fait que ce thème est susceptible d'approches multiples : histoire des institutions scolaires, histoire de la presse de l'éducation et de l'enseignement, histoire de l'éducation de la femme, histoire des disciplines et des manuels scolaires, histoire de l'alphabétisation, histoire de la lecture.

30 M. H. Camara Bastos, "História da educação do Rio Grande do Sul: estado da arte " in M. H. Camara Bastos, E. Tambara, L. Kreutz (dir.), Histórias e Memórias da Educação do Rio Grande do Sul, Pelotas, Publicações Seiva, 2002, p. 11-42.

31 Les Congressos Brasileiros de História da Educação (2000, 2002 et 2004), les Luso-brasileiros (1996, 1998, 2000, 2002 et 2004), les Iberoamericanos (1993, 1995, 1997, 1999, 2001 et 2003) et les Seminários HISTEDBR (1991, 1992, 1995, 1997, 2001, 2003, 2004, 2005).

32 Le Centre de documentation et de soutien à la recherche en histoire de l'éducation (Université de São Francisco), Mémoire de l'éducation et documents sur l'éducation brésilienne (chez les Auteurs associés), la série "Classiques de l'histoire et de la philosophie de l'éducation " (chez Seiva Publicações, Pelotas, RS). 
Quant aux bilans de la production historiographique, ils ont d'abord privilégié une approche nationale, dont diverses synthèses ont permis la critique ${ }^{33}$. Le grand risque de cette histoire nationale, macroscopique et généraliste, est qu'elle soit écrite à la lumière des États les plus influents et fasse voir la réalité brésilienne à travers le prisme de la région Sud-Est, comme cela a été le cas pour d'autres aspects de l'histoire du Brésil au XX' siècle, alors que les études régionales donnent une meilleure idée des écarts de développement

33 Une critique interne de la production a été réalisée par Miriam Warde (" Anotações para uma Historiografia da Educação Brasileira ", Em Aberto, Brasília, ann. 3, n 23, septembre/août 1984, p. 1-6 ; "Contribuições da História para a Educação ", Em Aberto, Brasília, v. 9, n 47, juillet/ septembre 1990, p. 3-11 ; "Questões teóricas e de Método: a História da Educação nos marcos de uma História das Disciplinas " in D. Saviani, J. Lombardi, J. Sanfelice (dir.), História e História da Educação. O debate teórico-metodológico atual, São Paulo, Autores Associados, 1998. p. 88-99 ; Clarice Nunes, "Locus da Produção da História da Educação hoje " in Rogério Fernandes, Áurea Adão (dir.), Leitura e escrita em Portugal e no Brasil (1500-1970), Porto, Sociedade Portuguesa de Ciências da Educação, 1998, vol. III, p. 579-588 ; Luiz Carlos Barreira, História e Historiografia: as escritas recentes da História da Educação Brasileira, Campinas, UNICAMP, 1995 (sa thèse) ; Leonor M. Tanuri, " Historiografia da Educação Brasileira: Contribuição para o seu estudo na década anterior à instalação dos cursos de Pós-Graduação ", História da Educação, ASPHE, Pelotas, vol. 2, n 3, avril 1998, p. 139-153 ; Elomar Tambara, "Problemas teórico-metodológicos da História da Educação " in D. Saviani, J. Lombardi, J. Sanfelice (dir.), História e História da Educação. O debate ..., op. cit., p. 79-87 ; Marta Maria Chagas de Carvalho, "A Configuração da Historiografia Educacional Brasileira " in Marcos de Freitas (dir.), Historiografia Brasileira em Perspectiva, São Paulo, Contexto, 1998, p. 329-353 ; ead., "L'histoire de l'éducation au Brésil : traditions historiographiques et processus de rénovation de la discipline ", Paedagogica historica, International Journal of the History of Education, vol. 36, n 3, 2000, p. 909-933 ; Bruno Bontempi Jr., "A historiografia da educação em Portugal e no Brasil: convergências e desafios ", Contemporaneidade e Educação, São Paulo, Ve an., $\mathrm{n}^{\circ}$ 7, 1er semestre 2000, p. 34-50 ; M. H.Camara Bastos, Marcus Levy, Albino Bencostta, Maria Teresa Santos Cunha, Uma cartografia da pesquisa em História da Educação na Região Sul: Paraná, Santa Catarina, Rio Grande do Sul (1980-2000), Pelotas, Publicações Seiva, 2004 ; Diana Gonçalves Vidal, Luciano Mendes de Faria Filho, "História da Educação no Brasil: a constituição histórica do campo (1880-1970) ", Revista Brasileira de História, ANPUH/Humanitas, vol. 23, 45, 2003, p. 37-70 ; Marta Maria de Araújo, "Tempo de balanço: a organização do campo educacional e a produção histórico-educacional brasileira e da região nordeste ", Revista Brasileira de História da Educação, SBHE/Campinas, $n^{\circ} 5$, janvier/juillet 2003, p. 9-41. D’autres études centrées sur certains espaces de production ont été présentées dans les congrès luso-brésiliens : voir Cláudia Costa Alves, "Os resumos das comunicações e as possibilidades esboçadas no II Congresso Luso-Brasileiro de História da Educação " in C. Sousa, D. Catani (dir.), Práticas educativas, culturas escolares, profissão docente, São Paulo, Escrituras, 1998 ; dans le GT Histoire de l'éducation, voir Denice B. Catani, Luciano M. de. Faria Filho, Um lugar de Produção a produção de um lugar. História e historiografia da educação Brasileira nos anos 80 e 90 - a produção divulgada no GT História da Educação, Caxambu, ANPED, 2001 ; dans le $1^{\text {er }}$ Congrès brésilien sur l'histoire de l'éducation, voir Libânea Nacif Xavier, "Particularidades de um campo disciplinar em consolidação: balanço do I Congresso Brasileiro de História da Educação (RJ/2000) » in SBHE (dir.), Educação no Brasil, Campinas, SBHE/Autores Associados ; dans les périodiques spécialisés, dans ce domaine voir M. H. Camara Bastos, Marcus Levy Albino Bencostta, Maria Teresa Santos Cunha, Uma cartografia da pesquisa..., op. cit. Par thématique : sur la culture scolaire, voir Rosa de Fátima Souza, "Um itinerário de pesquisa sobre cultura escolar " in Marcus V. Cunha (dir.) Ideário e imagens da educação escolar, Campinas, Autores Associados, 2001 ; sur les groupes scolaires, voir Luciano Faria Filho, Rosa Fátima Souza, "A contribuição dos estudos sobre Grupos Escolares para a renovação da história do ensino primário no Brasil » in Diana G. Vidal (dir.), Grupos Escolares: cultura escolar primária e escolarização da infância no Brasil (1893-1971), Campinas, Mercado das Letras, 2006, p. 21-56. 
et de la variété des situations dans le pays. Le grand tournant des années récentes a donc été de revenir aux archives et études régionales, grâce aux mémoires de maîtrise et thèses de doctorat portant sur des territoires plus circonscrits.

Le groupe de travail " Histoire de l'éducation " de l'ANPEd a cartographié la production historiographique par régions (Nordeste et Nord, Centre-ouest, Sud, Nordeste), mais aussi pour certains États (Minas Gerais ; São Paulo ; Rio de Janeiro ; Rio Grande do Sul ; Paraná ; Santa Catarina ; Ceará ; Sergipe). Venant à la suite de la parution de plusieurs études sur la question ${ }^{34}$ un séminaire sur la production de la recherche en histoire de l'éducation au Brésil a été organisé à Rio de Janeiro en août 2004. On a ainsi pu constater l'extraordinaire dispersion des thèmes et des approches, théoriques et méthodologiques, qu'on peut attribuer à la diversité de formation des chercheurs. Ceux-ci viennent en effet de divers horizons du champ éducatif et/ou de divers secteurs de la recherche en éducation, même si la part des historiens de formation augmente ${ }^{35}$.

C'est que l'histoire de la formation des maitres est en interférence permanente avec les recherches contemporaines sur la formation, dont chacun mesure les enjeux politiques et sociaux. Si le thème de la formation des maîtres est présent dans la majorité des programmes de pós-graduação en éducation ${ }^{36}$, le répertoire des groupes de recherche au Brésil (CNPQ, 2002), qui recense 350 groupes à la rubrique "formation des professeurs ", n'en recense plus que 28 à la rubrique " histoire de la formation des maîtres " et 8 à " histoire de la formation enseignante ", et les responsables des groupes de recherche ne sont pas des historiens mais appartiennent aux spécialités qui gravitent autour de la recherche en éducation. Quant au groupe de travail de l'ANPEd consacré à la formation des professeurs, il débat de l'actualité, discutant les problèmes posés par la formation initiale, par les cours de formation des professeurs d'école au

34 En 1987, l'INEP avait publié une étude intitulée " La Formation des professeurs au Brésil (1960-1980) "; voir aussi Marli André, O estado do conhecimento sobre a formação de professores no Brasil, 1990-1998, 2002.

Voir Dario Ragazzini, " Os estudos histórico-educativos e a História da Educação " in J. Sanfelice, D. Saviani, J. Lombardi (dir.), História da Educação. Perspectivas de um intercâmbio internacional, São Paulo, Autores Associados/HISTEDBR, 1999, ainsi que Diana Gonçalves Vidal, Luciano Mendes de Faria Filho, "História da Educação no Brasil: a constituição histórica do campo (1880-1970) ", Revista Brasileira de História, ANPUH/Humanitas, vol. 23, 45, 2003. p. 37-70.

36 Un forum privilégié de débats sur la formation des professeurs est l'Association nationale de formation des professionnels de l'éducation (ANFOPE) et ses associations régionales. 
niveau supérieur, par les pratiques innovantes. Dans le corpus de mémoires et de thèses concernant la formation des professeurs, sur un total de 284 titres, 21 études seulement ( 8 \%) avaient un caractère historique.

D'où des lacunes historiographiques importantes. Selon l'étude sur l'état des connaissances sur la formation des maîtres au Brésil publiée par Marli André en $2002^{37}$, celles-ci proviennent prioritairement de la région sud-est (Minas Gerais, Rio de Janeiro, São Paulo) ; de plus, autant les études sont nombreuses sur la formation des enseignants de l'école obligatoire, autant on observe " un silence presque total quant à la formation du professeur de l'enseignement supérieur, la formation pour les jeunes et les adultes, l'enseignement technique et agricole, l'éducation surveillée. Les sources analysées manifestent un excès de discours sur le thème de la formation enseignante et une insuffisance de données concrètes sur les pratiques et les politiques éducatives".

\section{2 - La formation des maîtres dans l'histoire de l'éducation}

On peut cependant se livrer à quelques considérations sur la situation actuelle de la recherche sur l'histoire de la formation enseignante.

En termes de périodisation, l'intérêt pour les recherches en histoire de l'éducation pendant la période coloniale (1500-1822) est encore limité, avec une préférence pour l'action éducative de la Compagnie de Jésus. Peu de recherches sont encore menées sur les autres congrégations enseignantes (franciscains, dominicains, carmélites, ursulines, etc.), dont le rôle a pourtant été décisif pour l'éducation brésilienne, avec l'arrivée de nouveaux ordres du milieu du XIX ${ }^{\mathrm{e}}$ siècle aux années 1930. La période des "Réformes de Pombal " (seconde moitié du XVIII siècle) porte surtout sur les réformes entreprises et sur les écoles royales (aulas régias), mais il n'y a pratiquement rien sur les maîtres d'écoles (professores régios), leur formation et leurs fonctions.

Concernant le $\mathrm{XIX}^{\mathrm{e}}$ siècle, un certain nombre de recherches portent sur la première école normale du Brésil, celle de la province de Rio de Janeiro (1868-1876), sur les processus de professionnalisation enseignante dans la province du Minas Gerais, la féminisation du professorat, des biographies de professeurs, les manuels scolaires. Mais la majorité des recherches portent sur le Brésil républicain. La Première République (1889-1930) concentre un grand nombre d'études, surtout sur les écoles complémentaires et les écoles normales créées pendant cette période. Aujourd'hui, l'historien de l'éducation 
se tourne vers la genèse et le développement du système républicain d'éducation. On observe également une préférence pour les études postérieures à 1960, qui font le bilan des réformes entreprises ; nombre de ces études font appel aux témoignages de professeurs sur leurs années de formation et d'exercice du métier.

Concernant la nature des cours de formation, à tous les niveaux, les recherches privilégient la formation du professeur du primaire dans les cours complémentaires et les cours normaux plutôt que la formation des professeurs dans les cours supérieurs (les licences et la pédagogie). L'histoire des écoles normales (histoire des institutions éducatives), des disciplines de formation (fondements de l'éducation et didactique), des lectures des professeurs font aussi l'objet de quelques recherches.

Les questions de genre, d'ethnie et de race bénéficient d'une attention croissante. À partir des années 1980, la question du genre entre dans la préoccupation de groupes travaillant sur l'histoire de l'éducation. Un nombre significatif d'études aborde la question de la féminisation du professorat, qui augmente avec l'expansion de l'enseignement primaire public à partir de la seconde moitié du XIX ${ }^{\mathrm{e}}$ siècle ; en revanche, peu de travaux portent sur la part des hommes dans la profession enseignante, concentrée dans l'enseignement moyen et supérieur et dans certaines disciplines. Ont aussi retenu l'attention des chercheurs les expériences de formation des professeurs dans des groupes immigrants depuis le $\mathrm{XIX}^{\mathrm{e}}$ siècle, spécialement dans les États du Sud, qui ont reçu d'importantes vagues d'immigrants allemands et italiens. On note également quelques études sur les trajectoires de formation de professeurs noirs, mais les recherches historiques sur ce thème n'en sont encore qu'à leur début. Même silence concernant la formation de professeurs indigènes, du fait qu'il s'agit d'une préoccupation politique récente. Selon les données du ministère de l'Éducation (2002), il existe 1392 écoles indigènes au Brésil, où enseignent 3059 professeurs indiens, et où étudient approximativement 93000 élèves, qui représentent 218 peuples dispersés dans l'ensemble du pays. Même si leur nombre n'est plus celui du début de la colonisation, nous avons aujourd'hui des exemples significatifs de la potentialité et de la singularité de chacun de ces peuples, qui parlent 180 idiomes différents et qui, progressivement, affichent leurs caractères culturels distinctifs. Traiter de l'histoire de l'éducation des peuples indigènes, c'est, sans doute, aborder l'histoire de chacun de ces groupes, avec 
ses particularités résultant de ses visions du monde ${ }^{38}$. C'est aussi mieux prendre en compte l'espace géographique brésilien, dans lequel la formation des professeurs ruraux a été longtemps ignorée au bénéfice des enseignants des grands centres urbains.

Avec la réforme du cours de pédagogie, diverses recherches portent plus spécifiquement sur la question de l'histoire de la formation des spécialistes, beaucoup étant centrées sur les mémoires de ces professionnels. Mais l'histoire des licences spécialisées est plus délaissée, sauf pour quelques disciplines, comme les mathématiques et l'histoire. Un nombre très réduit d'études concernent l'histoire de la formation des enseignants spécialisés œuvrant dans l'enseignement des handicapés, l'enseignement maternel, l'enseignement à distance. Enfin, un nombre significatif d'études historiques portent sur l'histoire de l'organisation des professeurs en associations et syndicats, surtout pendant la seconde moitié du $\mathrm{XX}^{\mathrm{e}}$ siècle. En revanche, la formation politique du professeur, ses conditions de travail, les formes d'association professionnelle, les questions salariales et de carrière sont des sujets encore peu étudiés ${ }^{39}$.

Ces diverses approches ont également conduit à un renouvellement de la réflexion sur l'exploitation des sources. Pour l'histoire de la formation des maîtres à l'époque récente, ce sont les sources orales qui sont privilégiées. L'histoire des grands acteurs de la formation repose classiquement sur les histoires de vie - biographies, autobiographies -, qui constituent un genre assez florissant. Enfin, on peut signaler le nombre et la qualité des recherches s'appuyant sur la presse d'enseignement et d'éducation, principalement celle du $\mathrm{XX}^{\mathrm{e}}$ siècle, alors que l'analyse des manuels didactiques tournés vers la formation enseignantes est encore en jachère.

À la fin de leur étude sur l'état actuel des connaissances sur la formation des enseignants, Brzezinski et Garrido ${ }^{40}$ se demandent : " Jusqu'à quel point

38 Maria Aparecida Bergamaschi, "Educação escolar indígena no século XX: da escola para os índios à escola específica e diferenciada " in M. Stephanou, M. H. Camara Bastos (dir.), Histórias e Memórias da Educação no Brasil: século XX, Petrópolis, Vozes, 2005.

39 Marli André (dir.), Formação de Professores no Brasil (1990-1998), Brasilia, MEC/INEp/Comped, 2002.

40 Iria Brzezinki, Elsa Garrido, "Análise dos trabalhos do GT Formação de Professores: o que revelam as pesquisas do período 1992-1998 ", Revista Brasileira de Educação, ANPEd, n 18, septembredécembre 2001, p. 82-100. 
les cours de formation des professeurs répondront-ils aux besoins de la société post-industrielle, de la révolution technologique, marquée par la production scientifique, par le développement des moyens de communication et d'information, par les inégalités et tensions sociales alarmantes, et par de nouvelles formes d'exercice de la citoyenneté ? Quels sont les nouveaux rôles du professeur? Quelle formation donner à l'éducateur pour qu'il puisse être un véritable agent de transformation de la société, intégré aux contextes culturels contemporains ? ". Parmi tant de réponses possibles, je dirai que la plus pertinente passe nécessairement par la connaissance historique des spécificités locales, régionales et nationales de la formation et de la profession enseignante au Brésil. Or, l'enseignement de l'histoire de l'éducation diminue dans les cursus de formation, en volume horaire et en contenu programmatique, au moment même où la recherche dans le domaine s'est considérablement développée. En conséquence, les historiens de l'éducation enseignent peu leur savoir dans les cours de graduação. Ainsi, il y a un hiatus entre la recherche, qui s'est développée en marge de l'enseignement, et les connaissances dispensées aux futurs enseignants ${ }^{41}$.

Un autre défi serait de constituer des groupes nationaux de recherche sur des thématiques et des objets communs à tout le Brésil : histoire des institutions scolaires, histoire de l'édition scolaire et éducative, histoire des disciplines scolaires, histoire des manuels, histoire de la formation enseignante. Ce serait la seule façon de réunir à un niveau macroscopique les données résultant d'études locales et régionales. L'histoire de l'éducation devrait également prendre en compte les cursus de pós-graduação en histoire, philosophie, psychologie, sociologie, disciplines qui sont considérées comme les sciences matrices des sciences de l'éducation.

Pour terminer, on observera que les recherches en histoire de l'éducation se sont orientées pour répondre aux questions concernant la consolidation d'un système public d'éducation. Il serait intéressant d'inverser cette formule et de se demander " à quel moment l'idée moderne de l'éducation se fonde au Brésil, et comment elle s'oriente d'abord vers sa propre connaissance puis sur

41 Un numéro de la Revista Brasileira de História da Educação (juillet/décembre 2003) comporte deux articles sur ce thème : Clarice Nunes, "O ensino da história da educação e a produção de sentidos na sala de aula " et F.L.M. Faria, J.R.G. Rodrigues, "A história da educação programada : uma aproximação da história da educação ensinada nos cursos de pedagogia em Belo Horizonte/MG ". 
la connaissance du système lui-même ". Comment et pourquoi l'histoire de l'éducation est-elle faite et écrite ${ }^{42}$ ?

Maria Helena CAMARA BASTOS

Pontificia Universidade Católica do Rio Grande do Sul, CNPq mhbastos@pucrs.br

42 Temístocles Cezar, "Presentismo, Memória e poesia. Noções da escrita da História do Brasil oitocentista " in Sandra J. Pesavento, Escrita, Linguagem, Objetos. Leituras de História Cultural, Bauru, EDUSC, 2004. p. 43-80, en part. p. 45. 